\title{
Chen-like inequalities on lightlike hypersurfaces of a Lorentzian manifold
}

\author{
Mehmet Gülbahar*, Erol Kilic and Sadık Keleș
}

\section{"Correspondence:}

mehmet.gulbahar@inonu.edu.tr

Department of Mathematics,

Faculty of Arts and Sciences, İönü

University, Malatya, Turkey

\begin{abstract}
We introduce k-Ricci curvature and k-scalar curvature on lightlike hypersurfaces of a Lorentzian manifold. We establish some inequalities between the extrinsic scalar curvature and the intrinsic scalar curvature. Using these inequalities, we obtain some characterizations on lightlike hypersurfaces. We give some results with regard to curvature invariants and $S\left(n_{1}, \ldots, n_{k}\right)$-spaces for lightlike hypersurfaces of a Lorentzian manifold.
\end{abstract}

\section{Introduction}

In 1873, Schläfli conjectured that every Riemann manifold could be locally considered as a submanifold of an Euclidean space with sufficient high codimension. This was proven by Janet in [1], Cartan in [2]. Friedmann extended the theorem to semi-Riemannian manifolds in [3]. Chen gave a relation between the sectional curvature and the shape operator for an $n$-dimensional submanifold $M$ in a Riemannian space form $R^{m}(\bar{c})$ in [4] as follows:

$$
A_{N}>\frac{n-1}{n}(c-\bar{c}) I_{n}
$$

where $A_{N}$ is a shape operator of $M, c=\inf K \neq \bar{c}$ and $I_{n}$ is an identity map. Also, Chen established a sharp inequality between the main intrinsic curvatures (the sectional curvature and the scalar curvature) and the main extrinsic curvatures (the squared mean curvature) for a submanifold in a real space form $R^{m}(\bar{c})$ in [5] as follows:

For each unit tangent vector $X \in T_{p} M^{n}$,

$$
H^{2}(p) \geq \frac{4}{n^{2}}\{\operatorname{Ric}(X)-(n-1) \bar{c}\},
$$

where $H^{2}$ is the squared mean curvature and $\operatorname{Ric}(X)$ is Ricci curvature of $M^{n}$ at $X$.

In [6], Hong and Tripathi presented a general inequality for submanifolds of a Riemannian manifold by using (1.2). In [7], this inequality was named Chen-Ricci inequality by Tripathi.

In [8] and [9], Chen introduced a Riemannian invariant $\delta\left(n_{1}, \ldots, n_{k}\right)$ by

$$
\delta\left(n_{1}, \ldots, n_{k}\right)=\tau(p)-\inf \left\{\tau_{\pi_{1}}(p)+\cdots+\tau_{\pi_{k}}(p)\right\}
$$

where $\tau(p)$ is scalar curvature of $M, \tau_{\pi_{j}}(p)$ is $j$-scalar curvature, $\pi_{1}, \ldots, \pi_{k}$ run over all $k$ mutually orthogonal subspaces of $T_{p} M$ such that $\operatorname{dim} \pi_{j}=n_{j}, j=1, \ldots, k$. In [10], the authors

(c) 2013 Gülbahar et al.; licensee Springer. This is an Open Access article distributed under the terms of the Creative Commons Attribution License (http://creativecommons.org/licenses/by/2.0), which permits unrestricted use, distribution, and reproduction in any medium, provided the original work is properly cited. 
gave optimal relationships among invariant $\delta\left(n_{1}, \ldots, n_{k}\right)$, the intrinsic curvatures and the extrinsic curvatures.

Later, Chen and some authors found inequalities for submanifolds of different spaces. For example, these inequalities were studied at submanifolds of complex space forms in [11-13]. Contact versions of Chen inequalities and their applications were introduced in [7, 14-16]. In [17], Tripathi investigated these inequalities in curvature-like tensors. Furthermore, Haesen presented an optimal inequality for an $m$-dimensional Lorentzian manifold embedded as a hypersurface on an $(m+1)$-dimensional Ricci-flat space in [18]. The authors in [19] proved an inequality using the extrinsic and the intrinsic scalar curvature in a semi-Riemannian manifold. In [20], Chen introduced space-like submanifolds (Riemannian submanifolds) of a semi-Riemannian manifold.

As far as we know, there is no paper about Chen-like inequalities and curvature invariants in lightlike geometry. So, we introduce $k$-plane Ricci curvature and $k$-plane scalar curvature of a lightlike hypersurface of a Lorentzian manifold. Using these curvatures, we establish some inequalities and by means of these inequalities, we give some characterizations of a lightlike hypersurface on a Lorentzian manifold. Finally, we introduce the curvature invariant $\delta\left(n_{1}, \ldots, n_{k}\right)$ on lightlike hypersurfaces of a Lorentzian manifold.

\section{Preliminaries}

Let $(\widetilde{M}, \widetilde{g})$ be an $(n+2)$-dimensional semi-Riemannian manifold and $M$ be a lightlike hypersurface of $\widetilde{M}$. The radical space or the null space of $T_{p} M$, at each point $p \in M$, is a one-dimensional subspace $\operatorname{Rad} T_{p} M$ defined by

$$
\operatorname{Rad} T_{p} M=\left\{\xi \in T_{p} M: g_{p}(\xi, X)=0, \forall X \in T_{p} M\right\}
$$

The complementary vector bundle $S(T M)$ of $\operatorname{Rad} T M$ in $T M$ is called the screen bundle of $M$. We note that any screen bundle is non-degenerate. Thus, we have

$$
T M=\operatorname{Rad} T M \perp S(T M),
$$

where $\perp$ denotes the orthogonal direct sum. The complementary vector bundle $S(T M)^{\perp}$ of $S(T M)$ is called screen transversal bundle and it has rank 2. Since Rad TM is a lightlike subbundle of $S(T M)^{\perp}$, there exists a unique local section $N$ of $S(T M)^{\perp}$ such that

$$
\widetilde{g}(N, N)=0, \quad \widetilde{g}(N, \xi)=1 .
$$

The Gauss and Weingarten formulas are given, respectively, by

$$
\begin{aligned}
& \widetilde{\nabla}_{X} Y=\nabla_{X} Y+h(X, Y), \\
& \widetilde{\nabla}_{X} N=-A_{N} X+\nabla_{X}^{t} N
\end{aligned}
$$

for any $X, Y \in \Gamma(T M)$, where $\nabla_{X} Y, A_{N} X \in \Gamma(T M)$ and $h(X, Y), \nabla_{X}^{t} N \in \Gamma(\operatorname{ltr}(T M))$. If we put $B(X, Y)=\widetilde{g}(h(X, Y), \xi)$ and $\tau(X)=\widetilde{g}\left(\nabla_{X}^{t} N, \xi\right)$, then (2.4) become

$$
\begin{aligned}
& \widetilde{\nabla}_{X} Y=\nabla_{X} Y+B(X, Y) N, \\
& \widetilde{\nabla}_{X} N=-A_{N} X+\tau(X) N,
\end{aligned}
$$


where $B$ and $A_{N}$ are called the second fundamental form and the shape operator of the lightlike hypersurface $M$. The induced connection $\nabla$ on $M$ is not metric connection but $\nabla$ is torsion free [21].

If $B=0$, then the lightlike hypersurface $M$ is called totally geodesic in $\widetilde{M}$. A point $p \in M$ is said to be umbilical if

$$
B(X, Y)_{p}=H g_{p}(X, Y), \quad \forall X, Y \in T_{p} M,
$$

where $H \in R$. $M$ is called totally umbilical in $\widetilde{M}$ if every point of $M$ is umbilical [21].

The mean curvature $\mu$ of $M$ with respect to an $\left\{e_{1}, \ldots, e_{n}\right\}$ orthonormal basis of $\Gamma(S(T M))$ is defined in [22] as follows:

$$
\mu=\frac{\operatorname{tr}(B)}{n}=\frac{1}{n} \sum_{i=1}^{n} \varepsilon_{i} B\left(e_{i}, e_{i}\right), \quad g\left(e_{i}, e_{i}\right)=\varepsilon_{i} .
$$

Let $P$ be a projection of $S(T M)$ on $M$. From (2.2), we have

$$
\begin{aligned}
\nabla_{X} P Y & =\nabla_{X}^{*} P Y+h(X, P Y) \\
& =\nabla_{X}^{*} Y+C(X, P Y) \xi, \quad X, Y \in \Gamma(T M), \\
\nabla_{X} \xi & =-A_{\xi}^{*} X-\tau(X) \xi
\end{aligned}
$$

where $\nabla_{X}^{*} P Y$ and $A_{\xi}^{*} X$ belong to $\Gamma(S(T M))$. Here $\nabla^{*}, C$ and $A_{\xi}^{*}$ are called the induced connection, the local second fundamental form and the local shape operator on $S(T M)$, respectively.

From (2.5) and (2.7) one has

$$
\begin{aligned}
& B(X, Y)=g\left(A_{\xi}^{*} X, Y\right), \\
& C(X, P Y)=g\left(A_{N} X, P Y\right) .
\end{aligned}
$$

Using (2.9) we have

$$
B(X, \xi)=0, \quad X \in \Gamma\left(\left.T M\right|_{U}\right)
$$

A lightlike hypersurface $(M, g)$ of a semi-Riemannian manifold $(\widetilde{M}, \widetilde{g})$ is called screen locally conformal if the shape operators $A_{N}$ and $A_{\xi}^{*}$ of $M$ and $S(T M)$, respectively, are related by

$$
A_{N}=\varphi A_{\xi}^{*}
$$

where $\varphi$ is a non-vanishing smooth function on a neighborhood $U$ on $M$ [23]. In particular, $M$ is called screen homothetic if $\varphi$ is a non-zero constant.

We denote the Riemann curvature tensors of $\widetilde{M}$ and $M$ by $\widetilde{R}$ and $R$, respectively. The Gauss-Codazzi type equations for $M$ are given as follows:

$$
\begin{aligned}
\widetilde{g}(\widetilde{R}(X, Y) Z, P U)= & g(R(X, Y) Z, P U)+B(X, Z) C(Y, P U) \\
& -B(Y, Z) C(X, P U),
\end{aligned}
$$




$$
\begin{aligned}
\tilde{g}(\widetilde{R}(X, Y) Z, \xi)= & \left(\nabla_{X} B\right)(Y, Z)-\left(\nabla_{Y} B\right)(X, Z)+B(Y, Z) \tau(X)-B(X, Z) \tau(Y), \\
\tilde{g}(\widetilde{R}(X, Y) Z, N)= & g(R(X, Y) Z, N), \\
\tilde{g}(\widetilde{R}(X, Y) P Z, N)= & \left(\nabla_{X} C\right)(Y, P Z)-\left(\nabla_{Y} C\right)(X, P Z) \\
& +\tau(Y) C(X, P Z)-\tau(X) C(Y, P Z)
\end{aligned}
$$

for any $X, Y, Z, U \in \Gamma(T M)[21]$.

Let $p \in M$ and $\Pi=\operatorname{sp}\left\{e_{i}, e_{j}\right\}$ be a two-dimensional non-degenerate plane of $T_{p} M$. The number

$$
K_{i j}=\frac{g\left(R\left(e_{j}, e_{i}\right) e_{i}, e_{j}\right)}{g\left(e_{i}, e_{i}\right) g\left(e_{j}, e_{j}\right)-g\left(e_{i}, e_{j}\right)^{2}}
$$

is called the sectional curvature at $p \in M$. Since the screen second fundamental form $C$ is not symmetric, the sectional curvature $K_{i j}$ of a lightlike submanifold is not symmetric, that is, $K_{i j} \neq K_{j i}$.

Let $p \in M$ and $\xi$ be a null vector of $T_{p} M$. A plane $\Pi$ of $T_{p} M$ is called a null plane if it contains $\xi$ and $e_{i}$ such that $\widetilde{g}\left(\xi, e_{i}\right)=0$ and $\widetilde{g}\left(e_{i}, e_{i}\right) \neq 0$. The null sectional curvature of $\Pi$ is given in [24] as follows:

$$
K_{i}^{\text {null }}=\frac{g\left(R_{p}\left(e_{i}, \xi\right) \xi, e_{i}\right)}{g_{p}\left(e_{i}, e_{i}\right)} .
$$

The Ricci tensor $\widetilde{\text { Ric }}$ of $\widetilde{M}$ and the induced Ricci type tensor $R^{(0,2)}$ of $M$ are defined by

$$
\begin{aligned}
& \widetilde{\operatorname{Ric}}(X, Y)=\operatorname{trace}\{Z \rightarrow \widetilde{R}(Z, X) Y\}, \quad \forall X, Y \in \Gamma(T \widetilde{M}), \\
& R^{(0,2)}(X, Y)=\operatorname{trace}\{Z \rightarrow R(Z, X) Y\}, \quad \forall X, Y \in \Gamma(T M) .
\end{aligned}
$$

Let $\left\{e_{1}, \ldots, e_{n}\right\}$ be an orthonormal frame of $\Gamma(S(T M))$. In this case,

$$
R^{(0,2)}(X, Y)=\sum_{j=1}^{n} \varepsilon_{j} g\left(R\left(e_{j}, X\right) Y, e_{j}\right)+\tilde{g}(R(\xi, X) Y, N),
$$

where $\varepsilon_{j}$ denotes the causal character $(\mp 1)$ of a vector field $e_{j}$. Ricci curvature of a lightlike hypersurface is not symmetric. Thus, Einstein hypersurfaces are not defined on any lightlike hypersurface. If $M$ admits that an induced symmetric Ricci tensor Ric and Ricci tensor satisfy

$$
\operatorname{Ric}(X, Y)=k g(X, Y), \quad \forall X, Y \in \Gamma(T M)
$$

where $k$ is a constant, then $M$ is called an Einstein hypersurface [23].

Let $M$ be a lightlike hypersurface of a Lorentzian manifold $\widetilde{M}$, replacing $X$ by $\xi$ and using (2.12), (2.13) and (2.14)

$$
\begin{aligned}
R^{(0,2)}(\xi, \xi) & =\sum_{j=1}^{n} g\left(R\left(e_{j}, \xi\right) \xi, e_{j}\right)-\widetilde{g}(R(\xi, \xi) \xi, N) \\
& =\sum_{j=1}^{n} K_{j}^{\text {null }} .
\end{aligned}
$$


Thus, we have

$$
\sum_{i=1}^{n} R^{(0,2)}\left(e_{i}, e_{i}\right)=\sum_{i=1}^{n}\left\{\sum_{j=1}^{n} g\left(R\left(e_{j}, e_{i}\right) e_{i}, e_{j}\right)\right\}+\sum_{i=1}^{n} \tilde{g}\left(R\left(\xi, e_{i}\right) e_{i}, N\right) .
$$

Adding (2.19) and (2.20), we obtain a scalar $\tau$ given as follows [25]:

$$
\begin{aligned}
\tau & =R^{(0,2)}(\xi, \xi)+\sum_{i=1}^{n} R^{(0,2)}\left(e_{i}, e_{i}\right) \\
& =\sum_{i, j=1}^{n} K_{i j}+\sum_{i=1}^{n} K_{i}^{\text {null }}+K_{i N},
\end{aligned}
$$

where $K_{i N}=\widetilde{g}\left(R\left(\xi, e_{i}\right) e_{i}, N\right)$ for $i \in\{1, \ldots, n\}$.

\section{3 k-Ricci curvature and $k$-scalar curvature}

Let $M$ be an $(n+1)$-dimensional lightlike hypersurface of a Lorentzian manifold $\tilde{M}$ and let $\left\{e_{1}, \ldots, e_{n}, \xi\right\}$ be a basis of $\Gamma(T M)$ where $\left\{e_{1}, \ldots, e_{n}\right\}$ is an orthonormal basis of $\Gamma(S(T M))$. For $k \leq n$, we set $\pi_{k, \xi}=\operatorname{sp}\left\{e_{1}, \ldots, e_{k}, \xi\right\}$ is a $(k+1)$-dimensional degenerate plane section and $\pi_{k}=\operatorname{sp}\left\{e_{1}, \ldots, e_{k}\right\}$ is a $k$-dimensional non-degenerate plane section.

We say that $k$-degenerate Ricci curvature and $k$-Ricci curvature at unit vector $X \in$ $\Gamma(T M)$ are as follows:

$$
\begin{aligned}
& \operatorname{Ric}_{\pi_{k, \xi}}(X)=R^{(0,2)}(X, X)=\sum_{j=1}^{k} g\left(R\left(e_{j}, X\right) X, e_{j}\right)+\widetilde{g}(R(\xi, X) X, N), \\
& \operatorname{Ric}_{\pi_{k}}(X)=R^{(0,2)}(X, X)=\sum_{j=1}^{k} g\left(R\left(e_{j}, X\right) X, e_{j}\right),
\end{aligned}
$$

respectively. Furthermore, we say that $k$-degenerate scalar curvature and $k$-scalar curvature at $p \in M$ are as follows:

$$
\begin{aligned}
& \tau_{\pi_{k, \xi}}(p)=\sum_{i, j=1}^{k} K_{i j}+\sum_{i=1}^{k} K_{i}^{n u l l}+K_{i N}, \\
& \tau_{\pi_{k}}(p)=\sum_{i, j=1}^{k} K_{i j},
\end{aligned}
$$

respectively. For $k=2, \Pi_{1, \xi}=\operatorname{sp}\left\{e_{1}, \xi\right\}$, then we have

$$
\operatorname{Ric}_{\Pi_{1, \xi}}\left(e_{1}\right)=K_{1 N},
$$

and

$$
\tau_{\Pi_{2}}(p)=K_{1}^{\text {null }}+K_{1 N}
$$


For $k=n, \pi_{n}=\operatorname{sp}\left\{e_{1}, \ldots, e_{n}\right\}=\Gamma(S(T M))$, then

$$
\operatorname{Ric}_{S(T M)}\left(e_{1}\right)=\operatorname{Ric}_{\pi_{n}}\left(e_{1}\right)=\sum_{j=1}^{n} K_{1 j}=K_{12}+\cdots+K_{1 n},
$$

and

$$
\tau_{S(T M)}(p)=\sum_{i, j=1}^{n} K_{i j}
$$

We say that screen Ricci curvature and screen scalar curvature are $\operatorname{Ric}_{S(T M)}\left(e_{1}\right)$ and $\tau_{S(T M)}(p)$, respectively. From (2.12) we can write

$$
\tau_{S(T M)}(p)=\tilde{\tau}_{S(T M)}(p)+\sum_{i, j=1}^{n} B_{i i} C_{j j}-B_{i j} C_{j i}
$$

where $B_{i j}=B\left(e_{i}, e_{j}\right)$ and $C_{i j}=C\left(e_{i}, e_{j}\right)$ for $i, j \in\{1, \ldots, n\}$.

Also, the components of the second fundamental form $B$ and the screen second fundamental form $C$ satisfy

$$
\sum_{i, j=1}^{n} B_{i j} C_{j i}=\frac{1}{2}\left\{\sum_{i, j=1}^{n}\left(B_{i j}+C_{j i}\right)^{2}-\sum_{i, j=1}^{n}\left(B_{i j}\right)^{2}+\left(C_{j i}\right)^{2}\right\},
$$

and

$$
\sum_{i, j} B_{i i} C_{j j}=\frac{1}{2}\left\{\left(\sum_{i, j} B_{i i}+C_{j j}\right)^{2}-\left(\sum_{i} B_{i i}\right)^{2}-\left(\sum_{j} C_{j j}\right)^{2}\right\} .
$$

Theorem 3.1 Let $M$ be an $(n+1)$-dimensional lightlike hypersurface of a Lorentzian manifold $\tilde{M}$. Then:

(a)

$$
\tau_{S(T M)}(p) \leq \tilde{\tau}_{S(T M)}(p)+n \mu\left(\operatorname{trace} A_{N}\right)+\frac{1}{4} \sum_{i, j}\left(B_{i j}-C_{j i}\right)^{2} .
$$

The equality holds for all $p \in M$ if and only if either $M$ is a screen homothetic lightlike hypersurface with $\varphi=-1$ or $M$ is a totally geodesic lightlike hypersurface.

(b)

$$
\tau_{S(T M)}(p) \geq \tilde{\tau}_{S(T M)}(p)+n \mu\left(\operatorname{trace} A_{N}\right)-\frac{1}{2} \sum_{i, j}\left(B_{i j}+C_{j i}\right)^{2} .
$$

The equality holds for all $p \in M$ if and only if either $M$ is a screen homothetic lightlike hypersurface with $\varphi=1$ or $M$ is a totally geodesic lightlike hypersurface.

(c) The equalities case of (3.10) and (3.11) hold at $p \in M$ if and only if $p$ is a totally geodesic point. 
Proof Using (3.7) and (3.8), we get

$$
\tau_{S(T M)}(p)=\widetilde{\tau}_{S(T M)}(p)+\sum_{i, j=1}^{n} B_{i i} C_{j j}-\frac{1}{2} \sum_{i, j=1}^{n}\left(B_{i j}+C_{j i}\right)^{2}+\frac{1}{2} \sum_{i, j=1}^{n}\left(B_{i j}\right)^{2}+\left(C_{j i}\right)^{2} .
$$

Since

$$
\frac{1}{2}\left(B_{i j}^{2}+C_{j i}^{2}\right)=\frac{1}{4}\left(B_{i j}+C_{j i}\right)^{2}+\frac{1}{4}\left(B_{i j}-C_{j i}\right)^{2},
$$

then

$$
\begin{aligned}
& \frac{1}{2}\left\{\sum_{i, j=1}^{n}\left(B_{i j}+C_{j i}\right)^{2}+\sum_{i, j=1}^{n}\left(B_{i j}\right)^{2}+\left(C_{j i}\right)^{2}\right\} \\
& =-\frac{1}{2} \sum_{i, j}\left(B_{i j}+C_{j i}\right)^{2}+\frac{1}{4} \sum_{i, j}\left(B_{i j}-C_{j i}\right)^{2} .
\end{aligned}
$$

If we put (3.13) in (3.12), we obtain

$$
\tau_{S(T M)}(p)=\widetilde{\tau}_{S(T M)}(p)+\sum_{i, j=1}^{n} B_{i i} C_{j j}-\frac{1}{2} \sum_{i, j}\left(B_{i j}+C_{j i}\right)^{2}+\frac{1}{4} \sum_{i, j}\left(B_{i j}-C_{j i}\right)^{2},
$$

which yields (3.10) and (3.11). From (3.10), (3.11) and (3.14) it is easy to get (a), (b) and (c) statements.

Corollary 3.2 Let M be an $(n+1)$-dimensional lightlike hypersurface of a Lorentzian space form $\widetilde{M}(c)$. Then:

(a)

$$
\tau_{S(T M)}(p) \leq n(n-1) c+n \mu\left(\operatorname{trace} A_{N}\right)+\frac{1}{4} \sum_{i, j}\left(B_{i j}-C_{j i}\right)^{2} .
$$

(b)

$$
\tau_{S(T M)}(p) \geq n(n-1) c+n \mu\left(\operatorname{trace} A_{N}\right)-\frac{1}{2} \sum_{i, j}\left(B_{i j}+C_{j i}\right)^{2}
$$

Corollary 3.3 Let $M$ be an $(n+1)$-dimensional screen homothetic lightlike hypersurface of a Lorentzian space form $\widetilde{M}(c)$. Then:

(a)

$$
\tau_{S(T M)}(p) \leq n(n-1) c+\varphi n^{2} \mu^{2}+\frac{(\varphi-1)}{4} \sum_{i, j}\left(B_{i j}\right)^{2} .
$$

(b)

$$
\tau_{S(T M)}(p) \geq n(n-1) c+\varphi n^{2} \mu^{2}-\frac{(\varphi-1)}{2} \sum_{i, j}\left(B_{i j}\right)^{2} .
$$


Theorem 3.4 Let $M$ be an $(n+1)$-dimensional lightlike hypersurface of a Lorentzian manifold $\widetilde{M}$. Then

$$
\begin{aligned}
\tau_{S(T M)}(p) \leq & \tilde{\tau}_{S(T M)}(p)+\frac{1}{2}(\operatorname{trace} \bar{A})^{2}-\frac{1}{2}\left(\operatorname{trace} A_{N}\right)^{2} \\
& -\frac{1}{2} \sum_{i, j}\left(B_{i j}+C_{j i}\right)^{2}+\frac{1}{4} \sum_{i, j}\left(B_{i j}-C_{j i}\right)^{2},
\end{aligned}
$$

where

$$
\bar{A}=\left(\begin{array}{cccc}
B_{11}+C_{11} & B_{12}+C_{21} & \cdots & B_{1 n}+C_{n 1} \\
B_{21}+C_{12} & B_{22}+C_{22} & \cdots & B_{2 n}+C_{n 2} \\
\vdots & & & \\
B_{n 1}+C_{1 n} & B_{n 2}+C_{2 n} & \cdots & B_{n n}+C_{n n}
\end{array}\right) .
$$

The equality of (3.19) holds for all $p \in M$ if and only if $M$ is minimal.

Proof From (3.14) and (3.9) we get

$$
\begin{aligned}
\tau_{S(T M)}(p)= & \widetilde{\tau}_{S(T M)}(p)+\frac{1}{2}\left\{\left(\sum_{i, j} B_{i i}+C_{j j}\right)^{2}-\left(\sum_{i} B_{i i}\right)^{2}-\left(\sum_{j} C_{j j}\right)^{2}\right\} \\
& -\frac{1}{2} \sum_{i, j}\left(B_{i j}+C_{j i}\right)^{2}+\frac{1}{4} \sum_{i, j}\left(B_{i j}-C_{j i}\right)^{2},
\end{aligned}
$$

which implies (3.19).

The equality of (3.19) satisfies then

$$
\sum_{i} B_{i i}=0
$$

This shows that $M$ is minimal.

By Theorem 3.4 we get the following corollaries.

Corollary 3.5 Let M be an $(n+1)$-dimensional lightlike hypersurface of a Lorentzian space form $\widetilde{M}(c)$. Then

$$
\begin{aligned}
\tau_{S(T M)}(p) \leq & n(n-1) c+\frac{1}{2}(\operatorname{trace} \bar{A})^{2}-\frac{1}{2}\left(\operatorname{trace} A_{N}\right)^{2} \\
& -\frac{1}{2} \sum_{i, j}\left(B_{i j}+C_{j i}\right)^{2}+\frac{1}{4} \sum_{i, j}\left(B_{i j}-C_{j i}\right)^{2},
\end{aligned}
$$

where $\bar{A}$ is equal to (3.20). The equality of (3.23) holds for all $p \in M$ if and only if $M$ is minimal. 
Corollary 3.6 Let $M$ be an $(n+1)$-dimensional screen homothetic lightlike hypersurface of a Lorentzian manifold $\widetilde{M}$. Then

$$
\tau_{S(T M)}(p) \leq \tilde{\tau}_{S(T M)}(p)+\frac{(2 \varphi+1)}{2} n^{2} \mu^{2}-\frac{\left(\varphi^{2}+6 \varphi+1\right)}{4} \sum_{i, j}\left(B_{i j}\right)^{2} .
$$

The equality of (3.24) holds for all $p \in M$ if and only if $M$ is minimal.

Now, we shall need the following lemma.

Lemma 3.7 [26] If $a_{1}, \ldots, a_{n}$ are $n$-real numbers $(n>1)$, then

$$
\frac{1}{n}\left(\sum_{i=1}^{n} a_{i}\right)^{2} \leq \sum_{i=1}^{n} a_{i}^{2}
$$

with equality if and only if $a_{1}=\cdots=a_{n}$.

Theorem 3.8 Let $M$ be an $(n+1)$-dimensional $(n>1)$ lightlike hypersurface of a Lorentzian manifold $\widetilde{M}$. Then

$$
\begin{aligned}
\tau_{S(T M)}(p) \leq & \tilde{\tau}_{S(T M)}(p)+\frac{n-1}{2 n}(\operatorname{trace} \bar{A})^{2}-\frac{1}{2}\left\{\left(\operatorname{trace} A_{N}\right)^{2}+n^{2} \mu^{2}\right\} \\
& +\frac{1}{4} \sum_{i, j}\left(B_{i j}-C_{j i}\right)^{2}-\frac{1}{2} \sum_{i \neq j}\left(B_{i j}+C_{j i}\right)^{2},
\end{aligned}
$$

where $\bar{A}$ is equal to (3.20).

The equality case of (3.26) holds for all $p \in M$ if and only if $n \mu=-\operatorname{trace} A_{N}$.

Proof From (3.21)

$$
\begin{aligned}
\tau_{S(T M)}(p)= & \widetilde{\tau}_{S(T M)}(p)+\frac{1}{2}\left\{(\operatorname{trace} \bar{A})^{2}-\left(\operatorname{trace} A_{N}\right)^{2}-n^{2} \mu^{2}\right\}-\frac{1}{2} \sum_{i}\left(B_{i i}+C_{i i}\right)^{2} \\
& -\frac{1}{2} \sum_{i \neq j}\left(B_{i j}+C_{j i}\right)^{2}+\frac{1}{4} \sum_{i, j}\left(B_{i j}-C_{j i}\right)^{2} .
\end{aligned}
$$

Using Lemma 3.7 and equality (3.27), we have

$$
\begin{aligned}
\tau_{S(T M)}(p) \leq & \tilde{\tau}_{S(T M)}(p)+\frac{1}{2}\left\{(\operatorname{trace} \bar{A})^{2}-\left(\operatorname{trace} A_{N}\right)^{2}-n^{2} \mu^{2}\right\}-\frac{1}{2 n}\left(\sum_{i} B_{i i}+C_{i i}\right)^{2} \\
& -\frac{1}{2} \sum_{i \neq j}\left(B_{i j}+C_{j i}\right)^{2}+\frac{1}{4} \sum_{i, j}\left(B_{i j}-C_{j i}\right)^{2},
\end{aligned}
$$

which implies (3.26).

The equality case of (3.26) satisfies then

$$
B_{11}+C_{11}=\cdots=B_{n n}+C_{n n} .
$$


From (3.29) we get

$$
\begin{aligned}
& (1-n) B_{11}+B_{22}+\cdots+B_{n n}+(1-n) C_{11}+C_{22}+\cdots+C_{n n}=0, \\
& B_{11}+(1-n) B_{22}+\cdots+B_{n n}+C_{11}+(1-n) C_{22}+\cdots+C_{n n}=0, \\
& \vdots \\
& B_{11}+B_{22}+\cdots+(1-n) B_{n n}+C_{11}+C_{22}+\cdots+(1-n) C_{n n}=0 .
\end{aligned}
$$

By the above equations, we obtain

$$
(n-1)^{2} \cdot\left(\operatorname{trace} A_{N}+n \mu\right)=0
$$

Since $n \neq 1, n \mu=-\operatorname{trace} A_{N}$.

From Theorem 3.8 we get the following corollaries.

Corollary 3.9 Let $M$ be an $(n+1)$-dimensional $(n>1)$ lightlike hypersurface of a Lorentzian space form $\widetilde{M}(c)$. Then

$$
\begin{aligned}
\tau_{S(T M)}(p) \leq & n(n-1) c+\frac{n-1}{2 n}(\operatorname{trace} \bar{A})^{2}-\frac{1}{2}\left\{n^{2} \mu^{2}+\left(\operatorname{trace} A_{N}\right)^{2}\right\} \\
& +\frac{1}{4} \sum_{i, j}\left(B_{i j}-C_{j i}\right)^{2}-\frac{1}{2} \sum_{i \neq j}\left(B_{i j}+C_{j i}\right)^{2},
\end{aligned}
$$

where $\bar{A}$ is equal to (3.20).

The equality case of (3.31) holds for all $p \in M$ if and only if $n \mu=-\operatorname{trace} A_{N}$.

Corollary 3.10 Let $M$ be an $(n+1)$-dimensional $(n>1)$ screen homothetic lightlike hypersurface of a Lorentzian manifold $\tilde{M}$. Then

$$
\begin{aligned}
\tau_{S(T M)}(p) \leq & \tilde{\tau}_{S(T M)}(p)+\varphi n^{2} \mu^{2}-\frac{(\varphi+1)^{2}}{2} n \mu^{2} \\
& +\frac{(\varphi-1)^{2}}{4} \sum_{i}\left(B_{i i}\right)^{2}-\frac{\left(\varphi^{2}+6 \varphi+1\right)}{4} \sum_{i \neq j}\left(B_{i j}\right)^{2} .
\end{aligned}
$$

The equality case of (3.32) holds for all $p \in M$ if and only if either $\varphi=-1$ or $M$ is minimal.

\section{Curvature invariants on lightlike hypersurfaces}

Definition 4.1 For an integer $k \geq 0$, let $\mathcal{S}(n, k)$ be the finite set which consists of $k$-tuples $\left(n_{1}, \ldots, n_{k}\right)$ of integers $\geq 2$ satisfying $n_{1}<n$ and $n_{1}+\cdots+n_{k} \leq n$. Denote by $\mathcal{S}(n)$ the set of all unordered $k$-tuples with $k \geq 0$ for a fixed positive integer $n$.

For each $k$-tuple $\left(n_{1}, \ldots, n_{k}\right) \in \mathcal{S}(n)$, the two sequences of curvature invariants $S\left(n_{1}, \ldots\right.$, $\left.n_{k}\right)(p)$ and $\hat{S}\left(n_{1}, \ldots, n_{k}\right)(p)$ are defined by, respectively,

$$
\begin{aligned}
& S\left(n_{1}, \ldots, n_{k}\right)(p)=\inf \left\{\tau\left(\Pi_{n_{1}}\right)+\cdots+\tau\left(\Pi_{n_{k}}\right)\right\}, \\
& \hat{S}\left(n_{1}, \ldots, n_{k}\right)(p)=\sup \left\{\tau\left(\Pi_{n_{1}}\right)+\cdots+\tau\left(\Pi_{n_{k}}\right)\right\},
\end{aligned}
$$


where $\Pi_{n_{1}}, \ldots, \Pi_{n_{k}}$ are $k$-dimensional mutually orthogonal subspaces of $T_{p} M$ such that $\operatorname{dim} \Pi_{n_{j}}=n_{j}, j=1, \ldots, k$.

$$
\begin{aligned}
& \delta\left(n_{1}, \ldots, n_{k}\right)(p)=\tau(p)-S\left(n_{1}, \ldots, n_{k}\right)(p), \\
& \hat{\delta}\left(n_{1}, \ldots, n_{k}\right)(p)=\tau(p)-\hat{S}\left(n_{1}, \ldots, n_{k}\right)(p) .
\end{aligned}
$$

We call a lightlike hypersurface an $S\left(n_{1}, \ldots, n_{k}\right)$ space if it satisfies $S\left(n_{1}, \ldots, n_{k}\right)=\hat{S}\left(n_{1}, \ldots\right.$, $\left.n_{k}\right)$.

Theorem 4.2 Let $M$ be a lightlike hypersurface of an $(n+2)$-dimensional Lorentzian manifold $\widetilde{M}$. Then $M$ is an $S(n)$ space if and only if the scalar curvature of $M$ is constant.

Proof Let $\left\{e_{1}, \ldots, e_{n}\right\}$ be an orthonormal frame of $\Gamma(S(T M))$. Let us choose $n$-dimensional plane sections such that

$$
\begin{aligned}
& \pi_{n-1, \xi}^{1}=\operatorname{sp}\left\{e_{2}, \ldots, e_{n}, \xi\right\} \subset T_{p} M, \\
& \vdots \\
& \pi_{n-1, \xi}^{n}=\operatorname{sp}\left\{e_{1}, \ldots, e_{n-1}, \xi\right\} \subset T_{p} M, \\
& \pi_{n}=\operatorname{sp}\left\{e_{1}, \ldots, e_{n}\right\} \subset T_{p} M .
\end{aligned}
$$

Thus, from (3.3) and (3.4), we obtain

$$
\begin{aligned}
& \tau_{\pi_{n-1, \xi}^{1}}(p)=\sum_{i, j=2}^{n} K_{i j}+K_{i}^{\text {null }}+K_{i N}, \\
& \vdots \\
& \tau_{\pi_{n-1, \xi}^{n}}(p)=\sum_{i, j=1}^{n-1} K_{i j}+K_{i}^{\text {null }}+K_{i N}, \\
& \tau_{\pi_{n}}(p)=\sum_{i, j=1}^{n} K_{i j}=\tau_{S(T M)}(p) .
\end{aligned}
$$

If $M$ is an $S(n)$ space, then we can write

$$
\begin{aligned}
& \tau_{\pi_{n-1, \xi}^{1}}(p)=\tau(p)-\operatorname{Ric}\left(e_{1}\right)-K_{1}^{\text {null }}=c, \\
& \vdots \\
& \tau_{\pi_{n-1, \xi}^{n}}(p)=\tau(p)-\operatorname{Ric}\left(e_{n}\right)-K_{n}^{\text {null }}=c, \\
& \tau_{\pi_{n}}(p)=\tau(p)-\sum_{i=1}^{n} K_{i}^{\text {null }}+K_{i N}=c .
\end{aligned}
$$

Therefore, we have

$$
\operatorname{Ric}\left(e_{1}\right)+K_{1}^{\text {null }}=\cdots=\operatorname{Ric}\left(e_{n}\right)+K_{n}^{\text {null }}=\sum_{i=1}^{n} K_{i}^{\text {null }}+K_{i N} .
$$


From (4.1) we get

$$
\operatorname{Ric}_{S(T M)}\left(e_{1}\right)+\cdots+\operatorname{Ric}_{S(T M)}\left(e_{n}\right)=(n-1) \sum_{i=1}^{n} K_{i}^{n u l l}+K_{i N}
$$

Using (4.2) we have

$$
\tau_{S(T M)}(p)=(n-1)\left(\tau(p)-\tau_{S(T M)}(p)\right) .
$$

Thus, we obtain

$$
\tau(p)=\left(\frac{2-n}{1-n}\right) c
$$

which shows that $\tau(p)$ is constant, which completes the proof.

Remark 4.3 We note that if an $n$-dimensional non-degenerate manifold is an $S(n)$ space, then it is an Einstein space (see [10]). On the other hand, if a degenerate hypersurface of a lightlike hypersurface is an $S(n)$ space, then it has constant scalar curvature. Thus, the curvature invariants on degenerate submanifolds give different characterizations from the curvature invariants on non-degenerate submanifolds.

Keeping in view (4.2), we get the following corollary immediately.

Corollary 4.4 Let $M(c)$ be an n-dimensional lightlike hypersurface with constant sectional curvature c. $M(c)$ is an $S(n)$ space if and only if $\sum_{i=1}^{n} K_{i N}=0$.

Now, we prove the following.

Theorem 4.5 Let $M$ be a lightlike hypersurface of an $(n+2)$-dimensional Lorentzian manifold $\tilde{M}$. If $M$ is an $S(j)$ space for $2 \leq j<n$, then $M$ is also $S(j+1)$ space.

Proof For the proof of the theorem, we use the induction method. Firstly, we show that the claim of the theorem is true for $j=2$. Suppose that $M$ is an $S(2)$ space. Let us choose any two-dimensional plane sections of $T_{p} M$ as $\Pi_{1, \xi}^{1}=\operatorname{sp}\left\{e_{1}, \xi\right\}, \Pi_{2}=\left\{e_{1}, e_{2}\right\}, \Pi_{1, \xi}^{2}=\operatorname{sp}\left\{e_{2}, \xi\right\}$. In that case,

$$
\begin{aligned}
& \tau_{\Pi_{1, \xi}^{1}}(p)=K_{1}^{\text {null }}+K_{1 N}=c, \\
& \tau_{\Pi_{2}}(p)=K_{12}+K_{21}=c, \\
& \tau_{\Pi_{1, \xi}^{2}}(p)=K_{2}^{\text {null }}+K_{2 N}=c .
\end{aligned}
$$

Now, let us choose three-dimensional plane sections of $T_{p} M$ as $\pi_{3}=\operatorname{sp}\left\{e_{1}, e_{2}, e_{3}\right\}, \pi_{2, \xi}^{1}=$ $\operatorname{sp}\left\{e_{1}, e_{2}, \xi\right\}$. If we show that $\tau_{\pi_{2, \xi}^{1}}(p)=\tau_{\pi_{3}}(p)=$ constant, then $M$ is an $S(3)$-space

$$
\begin{aligned}
\tau_{\pi_{2, \xi}^{1}}(p) & =K_{12}+K_{21}+\sum_{i=1}^{2} K_{i}^{\text {null }}+K_{i N} \\
& =3 c,
\end{aligned}
$$


and

$$
\begin{aligned}
\tau_{\pi_{3}}(p) & =K_{12}+K_{21}+K_{13}+K_{31}+K_{23}+K_{32} \\
& =3 c .
\end{aligned}
$$

Therefore, $M$ is an $S(3)$ space.

Now, we show that the claim of the theorem is true for $n=k$.

Let us choose any $k$-dimensional plane sections of $T_{p} M$ as $\pi_{k-1, \xi}^{1}=\operatorname{sp}\left\{e_{2}, e_{3}, \ldots, e_{k}, \xi\right\}$, $\pi_{k-1, \xi}^{2}=\operatorname{sp}\left\{e_{1}, e_{3}, \ldots, e_{k}, \xi\right\}, \ldots, \pi_{k-1, \xi}^{k}=\operatorname{sp}\left\{e_{1}, e_{2}, \ldots, e_{k-1}, \xi\right\}, \pi_{k}=\operatorname{sp}\left\{e_{1}, e_{2}, \ldots, e_{k}\right\}$. Then

$$
\begin{aligned}
& \tau_{\pi_{k-1, \xi}^{1}}(p)=\sum_{i, j=2}^{k} K_{i j}+\sum_{i=2}^{k} K_{i}^{\text {null }}+K_{i N}, \\
& \vdots \\
& \tau_{\pi_{k-1, \xi}^{k}}(p)=\sum_{i, j=1}^{k-1} K_{i j}+\sum_{i=1}^{k-1} K_{i}^{\text {null }}+K_{i N}, \\
& \tau_{\pi_{k}}(p)=\sum_{i, j=1}^{k} K_{i j} .
\end{aligned}
$$

From the above equations, we have

$$
\sum_{i=1}^{k} K_{i}^{n u l l}+K_{i N}=\frac{2 c}{k-1} .
$$

Let us choose $(k+1)$-dimensional plane sections of $T_{p} M$ as $\pi_{k, \xi}=\operatorname{sp}\left\{e_{1}, \ldots, e_{k}, \xi\right\}, \pi_{k+1}=$ $\operatorname{sp}\left\{e_{1}, \ldots, e_{k}, e_{k+1}\right\}$, then

$$
\begin{aligned}
\tau_{\pi_{k, \xi}}(p) & =\sum_{i, j=1} K_{i j}+\sum_{i=1}^{k} K_{i}^{\text {null }}+K_{i N} \\
& =c+\frac{2 c}{k-1}=\left(\frac{k+1}{k-1}\right) c .
\end{aligned}
$$

Using in a similar way a special case $j=2$, we obtain

$$
\tau_{\pi_{k+1}}(p)=\left(\frac{k+1}{k-1}\right) c .
$$

From (4.4) and (4.5) $M$ is an $S(k+1)$ space.

Theorem 4.6 Let $M$ be a lightlike hypersurface of an $(n+2)$-dimensional Lorentzian manifold $\tilde{M}$. Let $\left\{e_{1}, \ldots, e_{n}, \xi\right\}$ be an orthonormal basis of $p \in M$. If $M$ is an $S(n-1)$ space, then $\operatorname{Ric}\left(e_{1}\right)=\cdots=\operatorname{Ric}\left(e_{n}\right)=$ constant and $K_{1}^{\text {null }}=\cdots=K_{n}^{\text {null }}$.

Proof Let $M$ be an $S(n-1)$ space and $\pi_{n-2, \xi}^{1}=\operatorname{sp}\left\{e_{2}, \ldots, e_{n-1}, \xi\right\}, \pi_{n-2, \xi}^{2}=\operatorname{sp}\left\{e_{1}, e_{3}, \ldots, e_{n-1}, \xi\right\}$, $\ldots, \pi_{n-2, \xi}^{n-1}=\operatorname{sp}\left\{e_{1}, e_{2}, \ldots, e_{n-2}, \xi\right\}, \pi_{n-1}=\operatorname{sp}\left\{e_{1}, e_{2}, \ldots, e_{n-1}\right\}$ be $(n-1)$-dimensional plane sec- 
tions of $T_{p} M$. Then

$$
\begin{aligned}
& \tau_{\pi_{n-2, \xi}^{1}}(p)=\tau(p)-\operatorname{Ric}\left(e_{1}\right)-\operatorname{Ric}\left(e_{n}\right)-K_{1}^{\text {null }}-K_{n}^{\text {null }}=c, \\
& \tau_{\pi_{n-2, \xi}^{2}}(p)=\tau(p)-\operatorname{Ric}\left(e_{2}\right)-\operatorname{Ric}\left(e_{n}\right)-K_{1}^{\text {null }}-K_{n}^{\text {null }}=c, \\
& \vdots \\
& \tau_{\pi_{n-2, \xi}^{n-1}}(p)=\tau(p)-\operatorname{Ric}\left(e_{n-1}\right)-\operatorname{Ric}\left(e_{n}\right)-K_{n-1}^{\text {null }}-K_{n}^{\text {null }}=c, \\
& \tau_{\pi_{n-1}}(p)=\tau(p)-\operatorname{Ric}\left(e_{n}\right)-\sum_{i=1}^{n} K_{i}^{\text {null }}+K_{i N}=c .
\end{aligned}
$$

If we sum the above equations side to side and take into consideration Theorem 4.5 , we have

$$
\operatorname{Ric}\left(e_{1}\right)+\cdots+\operatorname{Ric}\left(e_{n}\right)+(n-1) \operatorname{Ric}\left(e_{n}\right)+\sum_{i=1}^{n} K_{i}^{n u l l}+\sum_{i=1}^{n-1} K_{i}^{n u l l}+K_{i N}=\text { constant. }
$$

Therefore, we obtain

$$
\begin{aligned}
& \operatorname{Ric}_{S(T M)}\left(e_{1}\right)+\cdots+\operatorname{Ric}_{S(T M)}\left(e_{n}\right)+(n-1) \operatorname{Ric}\left(e_{n}\right)+\sum_{i=1}^{n} K_{i}^{\text {null }}+K_{i N}+\sum_{i=1}^{n-1} K_{i}^{\text {null }}+K_{i N} \\
& \quad=\text { constant. }
\end{aligned}
$$

Taking into account upper equations, we get

$$
\tau_{S(T M)}(p)+(n-1) \operatorname{Ric}\left(e_{n}\right)+\tau(p)-\tau_{S(T M)}(p)+\tau_{\pi_{n-1, \xi}}(p)-\tau_{\pi_{n-1}}(p)=\text { constant, }
$$

where $\pi_{n-1, \xi}=\operatorname{sp}\left\{e_{1}, \ldots, e_{n-1}, \xi\right\}$ and $\pi_{n-1}=\operatorname{sp}\left\{e_{1}, \ldots, e_{n-1}\right\}$. Using Theorem 4.2 and Theorem 4.5, we obtain $\operatorname{Ric}\left(e_{n}\right)=$ constant. In addition to this, from (4.1), Theorem 4.2 and Theorem 4.5, we have $K_{1}^{\text {null }}=\cdots=K_{n}^{\text {null }}$, which completes the proof of the theorem.

In [25], Duggal restricted a lightlike hypersurface $M$ (labeled by $M^{0}$ ) of genus zero with screen distribution $S(T M)^{0}$. He denoted this type of a lightlike hypersurface by $C\left[M^{0}\right]=$ $\left[\left(M^{0}, g^{0}, S(T M)^{0}\right)\right]$ a class of lightlike hypersurfaces of genus zero such that

(a) $M^{0}$ admits a canonical screen distribution $S(T M)^{0}$ that induces a canonical lightlike transversal vector bundle $N^{0}$,

(b) $M^{0}$ admits an induced symmetric Ricci tensor, denoted by Ric ${ }^{0}$.

From above information, we get the following theorem immediately.

Theorem 4.7 Let $M^{0}$, a member of $C\left[M^{0}\right]$, be an $(2 n+1)$-dimensional lightlike hypersurface of a Lorentzian manifold $\widetilde{M}$. If $M^{0}$ is an Einstein hypersurface, then

$$
\tau_{\pi_{n, \xi}}(p)-\tau_{\pi_{n, \xi}^{\perp}}(p)=\sum_{i=1}^{n} K_{i}^{\text {null }}-\sum_{i=n+1}^{2 n} K_{i}^{\text {null }}
$$

where $\pi_{n, \xi}$ is any $(n+1)$-dimensional null section of $T_{p} M^{0}$ and $\pi_{n, \xi}^{\perp}$ denotes the orthogonal complement $\pi_{n, \xi}$ in $T_{p} M^{0}$. 
Proof Let us choose an orthonormal basis $\left\{e_{1}, \ldots, e_{2 n}\right\}$ at $p$ such that $\pi_{n, \xi}$ is spanned by $\left\{e_{1}, \ldots, e_{n}, \xi\right\}$. If $M^{0}$ is an Einstein hypersurface, then the Ricci curvature of $M^{0}$ satisfies

$$
\operatorname{Ric}\left(e_{1}\right)+\cdots+\operatorname{Ric}\left(e_{n}\right)=\operatorname{Ric}\left(e_{n+1}\right)+\cdots+\operatorname{Ric}\left(e_{2 n}\right) .
$$

From (2.17) we have

$$
\sum_{i=1}^{n} \sum_{j=1}^{2 n} K_{i j}+\sum_{i=1}^{n} K_{i N}=\sum_{i=n+1}^{2 n} \sum_{j=1}^{2 n} K_{i j}+\sum_{i=n+1}^{2 n} K_{i N}
$$

so, we get

$$
\sum_{i, j=1}^{n} K_{i j}+\sum_{i=1}^{n} K_{i N}=\sum_{i, j=n+1}^{n} K_{i j}+\sum_{i=n+1}^{n} K_{i N}
$$

which is equivalent to (4.6).

Now, we introduce these invariants as some special cases, and we get interesting characterizations on lightlike hypersurfaces as follows.

Theorem 4.8 Let $M$ be an $S\left(n_{1}, n_{1}\right)$-space. Then:

(a) If $n_{1}=2$, then $M$ is an $S(3)$-space.

(b) If $n_{1} \neq 2$, then $M$ is not necessary an $S\left(n_{1}+1\right)$-space. If

$$
\sum_{i=1}^{n_{1}} K_{i}^{\text {null }}+K_{i N}=\text { constant }
$$

then $M$ is an $S\left(n_{1}+1\right)$-space.

Proof (a) $n_{1}=2$, let us choose any two-dimensional plane sections of $T_{p} M$ as $\Pi_{1, \xi}^{1}=$ $\operatorname{sp}\left\{e_{1}, \xi\right\}, \Pi_{1, \xi}^{2}=\operatorname{sp}\left\{e_{2}, \xi\right\}, \Pi_{2}=\operatorname{sp}\left\{e_{1}, e_{2}\right\}$. Then

$$
\begin{aligned}
& \tau_{\Pi_{1, \xi}^{1}}(p)=K_{1}^{\text {null }}+K_{1 N}, \\
& \tau_{\Pi_{1, \xi}^{2}}(p)=K_{2}^{\text {null }}+K_{2 N}, \\
& \tau_{\Pi_{2}}(p)=K_{12}+K_{21} .
\end{aligned}
$$

If $M$ is an $S(2,2)$ space, then

$$
\begin{aligned}
& \tau_{\Pi_{1, \xi}^{1}}(p)+\tau_{\Pi_{1, \xi}^{2}}(p)=\sum_{i=1}^{2} K_{i}^{\text {null }}+K_{i N}=c, \\
& \tau_{\Pi_{1, \xi}^{1}}(p)+\tau_{\Pi_{2}}(p)=K_{12}+K_{21}+K_{1}^{\text {null }}+K_{1 N}=c, \\
& \tau_{\Pi_{1, \xi}^{2}}(p)+\tau_{\Pi_{2}}(p)=K_{12}+K_{21}+K_{2}^{\text {null }}+K_{2 N}=c .
\end{aligned}
$$


From the above equations, we have

$$
\tau_{\pi_{2, \xi}}(p)=\frac{3 c}{2}
$$

where $\pi_{2, \xi}=\operatorname{sp}\left\{e_{1}, e_{2}, \xi\right\}$ is a three-dimensional null plane section of $T_{p} M$.

Now, let us choose any two-dimensional plane sections of $T_{p} M$ as $\Pi_{2}^{1}=\operatorname{sp}\left\{e_{1}, e_{2}\right\}, \Pi_{2}^{2}=$ $\operatorname{sp}\left\{e_{1}, e_{3}\right\}, \Pi_{2}^{3}=\operatorname{sp}\left\{e_{2}, e_{3}\right\}$. Since $M$ is an $S(2,2)$-space, we can write

$$
K_{12}+K_{21}+K_{13}+K_{31}+K_{23}+K_{32}+K_{12}+K_{21}+K_{13}+K_{31}+K_{23}+K_{32}=2 \tau\left(\pi_{3}\right)=3 c .
$$

Therefore,

$$
\tau\left(\pi_{4}\right)=\frac{3 c}{2}
$$

where $\pi_{3}=\operatorname{sp}\left\{e_{1}, e_{2}, e_{3}\right\}$ is a three-dimensional non-degenerate plane section of $T_{p} M$. From (4.7) and (4.8) we see that $M$ is an $S(3)$-space.

(b) We show that the claim of the theorem is true for $n_{1}=3$. Let us choose any three-dimensional plane section of $T_{p} M$ as $\pi_{2, \xi}^{1}=\operatorname{sp}\left\{e_{1}, e_{2}, \xi\right\}, \pi_{2, \xi}^{2}=\operatorname{sp}\left\{e_{2}, e_{3}, \xi\right\}, \pi_{2, \xi}^{3}=$ $\operatorname{sp}\left\{e_{1}, e_{3}, \xi\right\}$. If $M$ is an $S(3,3)$-space, then

$$
\begin{aligned}
3 c & =2\left(\tau_{\pi_{2, \xi}^{1}}(p)+\tau_{\pi_{2, \xi}^{2}}(p)+\tau_{\pi_{2, \xi}^{3}}(p)\right) \\
& =2 \tau_{\pi_{3, \xi}}(p)+2 \sum_{i=1}^{3} K_{i}^{\text {null }}+K_{i N},
\end{aligned}
$$

where $\pi_{3, \xi}=\operatorname{sp}\left\{e_{1}, e_{2}, e_{3}, \xi\right\} \subset T_{p} M$. Consider (4.9), we obtain the proof of (b) condition is true.

The proof of a general case has been seen using the same way as the special case $n_{1}=3$.

Theorem 4.9 Let $M$ be a $(2 n+1)$-dimensional lightlike hypersurface of a Lorentzian manifold $\tilde{M}$.

(a) If $\inf \left\{\tau\left(\pi_{n}\right)+\tau\left(\pi_{n+1}\right)\right\}>0$, then $\tau(p)>0$.

(b) If $\sup \left\{\tau\left(\pi_{n}\right)+\tau\left(\pi_{n+1}\right)\right\}<0$, then $\tau(p)<0$.

Proof Let $T_{p} M=\operatorname{sp}\left\{e_{1}, \ldots, e_{2 n}, \xi\right\}$. We suppose that $\inf \left\{\tau\left(\pi_{n}\right)+\tau\left(\pi_{n+1}\right)\right\}>0$. By straightforward computation, we have

$$
C(2 n-2, n-2)+C(2 n-2, n) \tau_{S(T M)}(p)+C(2 n-1, n-1) \sum_{i=1}^{2 n} K_{i}^{n u l l}+K_{i N}>0
$$

and

$$
C(2 n-2, n-3)+C(2 n-2, n-1) \tau_{S(T M)}(p)+C(2 n, n) \sum_{i=1}^{2 n} K_{i}^{n u l l}+K_{i N}>0
$$


Summing up (4.10) and (4.11), we get

$$
C(2 n, n) \tau_{S(T M)}(p)+C(2 n, n) \sum_{i=1}^{2 n} K_{i}^{n u l l}+K_{i N}>0,
$$

which shows that $C(2 n, n) \tau(p)>0$. Therefore, $\tau(p)>0$ which is a proof of the statement (a). Now, we suppose that $\sup \left\{\tau\left(\pi_{n}\right)+\tau\left(\pi_{n+1}\right)\right\}>0$. Following a similar way in the proof of statement (a), we have

$$
C(2 n, n) \tau_{S(T M)}(p)+C(2 n, n) \sum_{i=1}^{2 n} K_{i}^{n u l l}+K_{i N}<0,
$$

which shows that $C(2 n, n) \tau(p)<0$. Therefore $\tau(p)<0$, which is a proof of the statement (b).

\section{Competing interests}

The authors declare that they have no competing interests.

\section{Authors' contributions}

The authors did not provide this information.

\section{Acknowledgements}

The authors have greatly benefited from the referee's report. So we wish to express our gratitude to the reviewer for his/her valuable suggestions which improved the content and presentation of the paper.

Received: 31 December 2012 Accepted: 13 May 2013 Published: 28 May 2013

\section{References}

1. Janet, M: Sur la possibilité de plonger un espace Riemannian donné dans un espace Euclidien. Ann. Soc. Pol. Math. 5 , 38-43 (1926)

2. Cartan, E: Sur la possibilité de plonger un espace Riemannian donné dans un espace Euclidien. Ann. Soc. Pol. Math. 5, 1-7 (1927)

3. Friedman, A: Isometric embedding of Riemannian manifolds into Euclidean spaces. Rev. Mod. Phys. 37, 201-203 (1965)

4. Chen, BY: Mean curvature and shape operator of isometric immersions in real space forms. Glasg. Math. J. 38, 87-97 (1996)

5. Chen, BY: Relations between Ricci curvature and shape operator for submanifolds with arbitrary codimension. Glasg. Math. J. 41, 33-41 (1999)

6. Hong, S, Tripathi, MM: On Ricci curvature of submanifolds. Int. J. Pure Appl. Math. Sci. 2, 227-245 (2005)

7. Tripathi, MM: Chen-Ricci inequality for submanifolds of contact metric manifolds. J. Adv. Math. Stud. 1, 111-134 (2008)

8. Chen, BY: Some pinching and classification theorems for minimal submanifolds. Arch. Math. 60, 568-578 (1993)

9. Chen, BY: A Riemannian invariant and its applications to submanifold theory. Results Math. 27, 17-26 (1995)

10. Chen, BY, Dillen, F, Verstraelen, L, Vrancken, V: Characterizations of Riemannian space forms, Einstein spaces and conformally flat spaces. Proc. Am. Math. Soc. 128, 589-598 (2000)

11. Kim, JS, Song, YM, Tripathi, MM: Shape operator for slant submanifolds in generalized complex space form. Indian J. Pure Appl. Math. 34(8), 1153-1163 (2003)

12. Matsumoto, K, Mihai, I, Oiagǎ, A: Shape operator for slant submanifolds in complex space forms. Bull. Yamagata Univ. Nat. Sci. 14, 169-177 (2000)

13. Mihai, A: B.Y. Chen inequalities for slant submanifolds in generalized complex space forms. Rad. Mat. 12, 215-231 (2004)

14. Cioroboiu, D, Oiagă, A: B.Y. Chen inequalities for slant submanifolds in Sasakian space forms. Rend. Circ. Mat. Palermo 52, 367-381 (2003)

15. Kim, YH, Lee, CW, Yoon, DW: Shape operator of slant submanifolds in Sasakian space forms. Bull. Korean Math. Soc. 40, 63-76 (2003)

16. Liu, X, Zhou, J: On Ricci curvature of certain submanifolds in a cosymplectic space form. Sarajevo J. Math. 2, 95-106 (2006)

17. Tripathi, MM: Improved Chen-Ricci inequality for curvature-like tensors and its applications. Differ. Geom. Appl. 29, 685-698 (2011)

18. Haesen, S: Optimal inequalities for embedded space-times. Kragujev. J. Math. 28, 69-85 (2005)

19. Dillen, F, Haesen, S, Miroslava, PT, Verstraelen, L: An inequality between intrinsic and extrinsic curvature invariants for codimension 2 embeddings. J. Geom. Phys. 52, 101-112 (2004)

20. Chen, BY: Pseudo-Riemannian Geometry, $\delta$-Invariants and Applications. World Scientific, Singapore (2011) 
21. Duggal, KL, Bejancu, A: Lightlike Submanifolds of Semi-Riemannian Manifolds and Applications. Kluwer Academic, Dordrecht (1996)

22. Bejan, CL, Duggal, KL: Global lightlike manifolds and harmonicity. Kodai Math. J. 28, 131-145 (2005)

23. Duggal, KL, Sahin, B: Differential Geometry of Lightlike Submanifolds. Birkhäuser, Basel (2010)

24. Beem, JK, Ehrlich, PE, Easley, KL: Global Lorentzian Geometry, 2nd edn. Dekker, New York (1996)

25. Duggal, KL: On scalar curvature in lightlike geometry. J. Geom. Phys. 57, 473-481 (2007)

26. Tripathi, MM: Certain basic inequalities for submanifolds in $(\kappa, \mu)$-space. In: Recent advances in Riemannian and Lorentzian geometries (Baltimore, MD), pp. 187-202 (2003)

doi:10.1186/1029-242X-2013-266

Cite this article as: Gülbahar et al.: Chen-like inequalities on lightlike hypersurfaces of a Lorentzian manifold. Journal of Inequalities and Applications 2013 2013:266.

Submit your manuscript to a SpringerOpen ${ }^{\odot}$ journal and benefit from:

- Convenient online submission

- Rigorous peer review

- Immediate publication on acceptance

- Open access: articles freely available online

- High visibility within the field

- Retaining the copyright to your article

Submit your next manuscript at $>$ springeropen.com 